\title{
Manejo y Reproducción de la Lechuza Mantequera (Tyto alba) en el Zoológico Metropolitano Rosy Walther, Honduras
}

\author{
Francisco Aceituno y Carmen Núñez ${ }^{2}$
}

\begin{abstract}
Resumen. En esta nota se presenta información relacionada con el manejo en cautiverio de la lechuza mantequera Tyto alba en el Zoológico Metropolitano Rosy Walther, Honduras. El objetivo fue documentar los datos más importantes sobre el comportamiento, alimentación y tamaño de los alojamientos. Además, durante el período comprendido entre diciembre 2013 y febrero 2014 se dio seguimiento al nacimiento de dos polluelos. El tamaño de puesta conocido de la especie en condiciones de cautiverio se redujo a dos huevos y el desarrollo de los pichones se completó hacia la doceava semana cuando desapareció el plumaje juvenil y se alimentaban por ellos mismos.
\end{abstract}

Palabras clave: Cautiverio, comportamiento, dieta, recinto.

Abstract. In this paper we report information related with the captive management of Barn Owl Tyto alba at the Metropolitan Zoo Rosy Walther, Honduras. The aim in this work was to record the most important data of the behavior, feeding, and the enclosure size. During the period of December 2013 to February 2014, the breeding of two young owls was monitored. The record of the clutch size in captivity was reduced to two eggs, and the growth of the chicks was completed by the twelfth week when the juvenile plumage disappeared and fed themselves.

Key words: Captivity, behavior, diet, enclosure.

\section{Introducción}

La lechuza mantequera (Tyto alba) está ampliamente distribuida en todo el mundo y se puede observar desde zonas templadas hasta zonas tropicales (Taylor, 1994). En Honduras fue reportada por primera vez en 1936, cuando se registró un nido en la isla de Guanaja, aquí se observó un juvenil y dos huevos (Bond, 1936). Monroe, en 1968, reportó 21 especímenes colectados para el país, destacando que la especie es bastante común.

Bonta y Anderson 2003 la señalan como una especie residente que se reproduce en el país, en áreas abiertas e intervenidas y de frecuencia bastante común. Los datos de la especie son relativamente abundantes y han sido documentados extensamente, sin embargo todavía falta información para comprender los factores que influyen en la capacidad de reproducción en diferentes ambientes (Marti, 1994).

El Zoológico Rosy Walther mantiene una colección de aves en exhibición, además recibe fauna silvestre que ha sido decomisada o donada por sus dueños, está localizado en el Cerro el Picacho de la ciudad de Tegucigalpa (14'12' 24" N; 87¹9'02" W), Honduras. El objetivo de la presente nota fue documentar las experiencias en el mantenimiento en cautiverio de los ejemplares de lechuza mantequera en el Zoológico Rosy Walther, esta información puede ser útil para considerar los principales aspectos para la supervivencia de la especie en condiciones ex situ.

\section{Comportamiento}

La mayoria de buhos y lechuzas presentan diferentes posturas cuando se sienten amenazados, en la lechuza mantequera se han registrado ciclos de movimientos de balanceo de la cabeza, acompañados con vocalizaciones de amenaza (Johnsgard, 1989). El zoológico cuenta con cuatro especímenes de Tyto alba y su comportamiento generalmente no es agresivo, a menos que los cuidadores se aproximen mucho a las cajas que utilizan como dormideros. En tal sentido, y no obstante que la especie es nocturna, presenta actividad diurna especialmente a la hora que se le alimenta.

\footnotetext{
${ }^{1}$ Sub Unidad de Biología y Veterinaria, Zoológico Rosy Walther, Tegucigalpa, Honduras. E-mail: aceitunof@yahoo.com

${ }^{2}$ Estudiante, Departamento de Biología, Facultad de Ciencias, Universidad Nacional Autónoma de Honduras, Tegucigalpa, Honduras. E-mail: julycar03@yahoo.es
} 


\section{Recinto}

Los especímenes pasan por un periodo de cuarentena de 30 días antes de introducirlos a la colección de exhibición, posteriormente son alojados en un recinto que tiene un área de $6 \mathrm{~m}$ de largo por 6 $\mathrm{m}$ de ancho. Dentro del recinto se utilizan cajas de madera de pino como dormideros (Figura 1), las cuales tienen una dimensión de $60 \mathrm{~cm}$ de ancho por 50 cm de altura, con un techo de lámina de zinc, y una percha interna; cada caja tiene una abertura de entrada en el frente en forma de arco, que mide $28 \mathrm{~cm}$ de largo por $21 \mathrm{~cm}$ de ancho y se encuentra a una altura de $1.5 \mathrm{~m}$ del suelo.

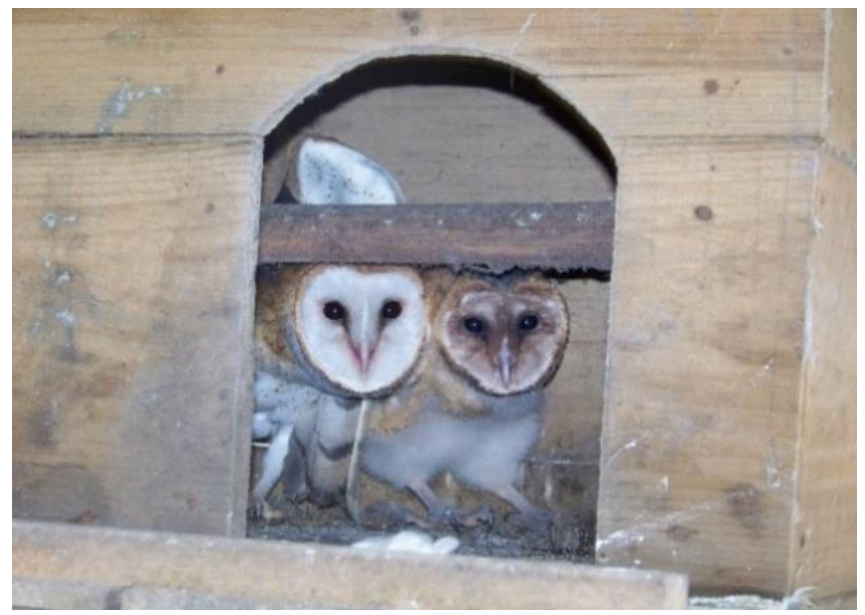

Figura.1. Pichón y su madre de la lechuza Tyto alba en caja dormidero en el Zoológico Metropolitano Rosy Walther, Honduras.

\section{Dieta}

En estudios realizados para analizar los restos no digeridos del contenido estomacal de la especie, se ha determinado que hasta un $98 \%$ de su alimentación se basa en roedores (Lenton, 1984). En el Zoológico, la especie se alimenta con ratones (Mus musculus) en caso de no contar con ratones se utiliza como substituto el pollo o carne de res. Se alimenta todos los días alrededor de las 11:00 de la mañana y se le proporciona en promedio tres ratones por individuo que se le colocan en una pequeña plataforma en la entrada de las cajas dormideros.

\section{Reproducción}

En la primera semana de diciembre del 2013 se halló un pichón en uno de los dormideros del recinto, posteriormente se descubrió un segundo pichón que había nacido en la misma nidada, el seguimiento del crecimiento se hizo mediante observaciones ad libitum, realizando avistamientos por la mañana y por la tarde de acuerdo a los periodos donde la lechuza presentaba más actividad.

No se encontró evidencias de la construcción de nido, esto concuerda con lo reportado por Nores y Gutiérrez (1986) los cuales indican que la especie se limita a depositar los huevos directamente sobre el suelo de las estructuras donde anida. En este caso no se encontraron rastros de otros huevos puestos, en contraste con las observaciones de Maestrelli (1973) que documentó tres huevos por nidada en condiciones de cautiverio. Esta disminución en el tamaño de la nidada puede estar relacionada con el tamaño de las presas, según Lenton (1984) este es un factor decisivo que afecta directamente el éxito reproductivo de las nidadas.

Lynch (2007) indica que las hembras hacen todo el proceso de incubación, que dura entre 29 y 34 días, y comienza justo después de haber puesto el primer o el segundo huevo. Las observaciones indican que durante las tres primeras semanas los pichones mantuvieron un plumón blanco en casi todo el cuerpo y hacia la sexta semana se observó que el disco facial presentaba plumas color canela y el plumón solo permanecía en la zona ventral y las patas, además, mantenían los ojos abiertos (Figura 2), como lo indican Nores y Gutiérrez (1986). Se observaron movimientos de la cabeza de izquierda a derecha, con el pico dirigido hacia las patas, ante la presencia de extraños.

Para la décima semana el plumón solo se observaba en las patas y en la doceava semana los pichones prácticamente habían perdido todo el plumón y presentan un plumaje similar al de los adultos, solamente diferenciándolos porque el disco facial muestra una coloración café obscura; ya realizaban intentos de vuelo moviéndose hacia otras cajas dentro del mismo recinto. Además, se observó que al colocar los ratones en la plataforma del dormidero uno de los adultos se encargaba de introducirlos en el nido; se 
puede suponer que era el padre el que realizaba la acción de recoger los ratones. Las lechuzas mantienen una división de roles y el padre es el encargado de llevar alimento al nido y la madre es la encargada de cuidar los pichones (Mikkola, 1983; Roulin y Bersier, 2007. Este patrón se mantuvo durante las doce semanas en las que se realizaron las observaciones, aunque en la semana siete se vio a uno de los pichones salir de la caja para alimentase.

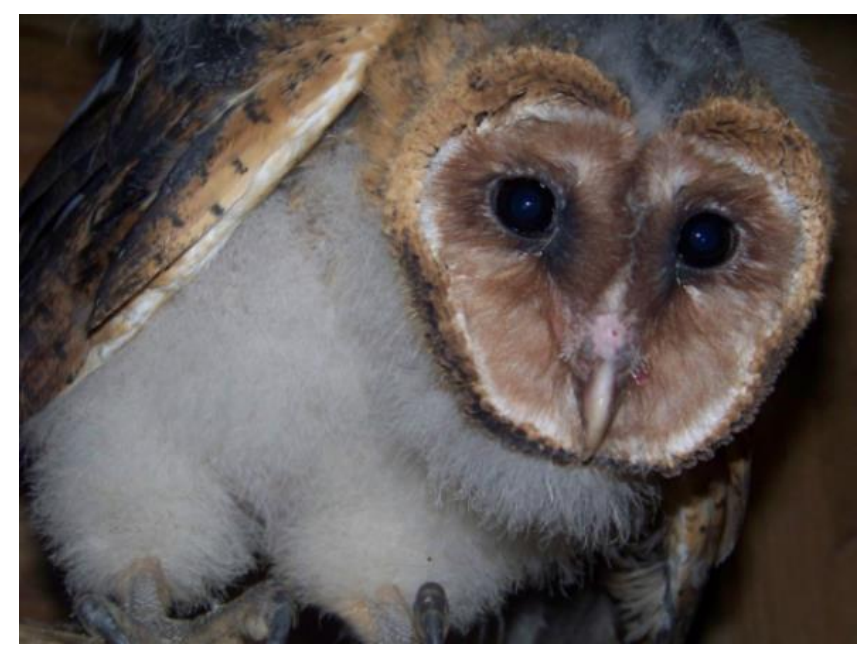

Figura 2. Pichón de Tyto alba de seis semanas de vida.

En el recinto, además de los padres y los pichones, se encontraba otra lechuza y un búho moteado (Ciccaba virgata), a pesar de esto no se observaron agresiones entre los individuos del recinto y los padres durante el desarrollo de los pichones; sin embargo hubo agresiones cuando se ingresó una nueva lechuza en el recinto la cual fue atacada provocándole la muerte.

Mendelssohn y Marder (1970) indican que el éxito reproductivo en cautiverio de las aves rapaces es relativamente más raro que en otros grupos de aves. Los datos aquí presentados contribuyen al conocimiento de la especie a nivel nacional y son útiles para mejorar su manejo en cautiverio.
Agradecimiento. Los autores quieren agradecer a la Dra. Sherry Thorn, a la Lic. Delmy Trochez y al Lic. Enmanuel Meraz por la revisión y comentarios a esta nota.

\section{Literatura Citada}

Bond, J. 1936. Resident birds of the Bay Islands of Spanish Honduras. Proceedings of the Academy of Natural Sciences of Philadelphia 88:353-364.

Bonta, M. y D.L. Anderson. 2003. Birding Honduras: A checklist and guide. Tegucigalpa, Honduras: Ecoarte. $186 \mathrm{p}$.

Johnsgard, P. 1989. Social Behavior of North American Owls: biology and natural history. Washington, DC: Smithsonian Institution Press. 295 p.

Lenton, G.M. 1984. The feeding and breeding ecology of Barn Owls Tyto alba in Peninsular Ma1aysia. Ibis 126:551-575.

Lynch, W. 2007. Owls of the United States and Canada: a complete guide to their biology and behavior. The Johns Hopkins University Press. 242 p.

Maestrelli, J.R. 1973. Propagation of Barn Owl in captivity. Auk 90:426-428.

Marti, C.D. 1994. Barn Owl reproduction: Patterns and variation near the limit of the species distribution. Condor 96:468-484.

Mendelssohn, H. y U. Marder. 1970. Problems of reproduction in birds of prey in captivity. International Zoo Yearbook 10 (1):6-11.

Mikkola, H. 1983. Owls in Europe. Calton: T. \& A. D. Poyser. 397 p.

Monroe, B.M., Jr. 1968. A distributional survey of the birds of Honduras. Ornithology Monographs 7:1-478.

Nores, A.I. y M. Gutiérrez. 1986. Nidificación de Tyto alba en Córdoba, Argentina. Hornero 12 (4):242-249.

Roulin, A. y L.F. Bersier. 2007. Nestling barn owls beg more intensely in the presence of their mother than in the presence of their father. Animal behavior 74:1099-1106.

Taylor, I. 1994. Barn owls: predator-prey relationships and conservation. Cambridge University Press. 304 p.

Recibido para publicación el 30 de julio de 2014.

Aceptado para publicación el 9 de diciembre de 2014. 